\title{
Measurement of double parton interactions in W+2 jets events with the CMS detector
}

\author{
Anastasia Grebenyuk* \\ IIHE- Université Libre de Bruxelles \\ E-mail: Anastasia.Grebenyuk@cern.ch
}

Double parton scattering is investigated in proton-proton collisions at $\sqrt{s}=7 \mathrm{TeV}$ where the final state includes a $\mathrm{W}$ boson, which decays into a muon and a neutrino, and two jets. The data sample corresponds to an integrated luminosity of $5 \mathrm{fb}^{-1}$, collected with the CMS detector at the LHC. Observables sensitive to double parton scattering are investigated after being corrected for detector effects and selection efficiencies. The fraction of $\mathrm{W}+2$-jet events due to double parton scattering is measured to be $0.055 \pm 0.002$ (stat.) \pm 0.014 (syst.). The effective cross section, $\sigma_{\text {eff }}$, characterizing the effective transverse area of hard partonic interactions in collisions between protons is measured to be $20.7 \pm 0.8$ (stat.) \pm 6.6 (syst.) $\mathrm{mb}$.

XXII. International Workshop on Deep-Inelastic Scattering and Related Subjects, 28 April - 2 May 2014

Warsaw, Poland

* On behalf of the CMS collaboration. 


\section{Introduction}

In high-energy proton-proton (pp) collisions at the Large Hadron Collider (LHC), semi-hard parton-parton scattering, producing particles with transverse momenta $p_{T}$ of a few $\mathrm{GeV}$, dominates the inelastic cross section. In such processes longitudinal momentum fractions, given by $x \sim 2 p_{T} / \sqrt{s}$, of values down to $\mathscr{O}\left(10^{-3}\right)$ are probed. At these values of $x$, the parton densities are large causing a sizable probability for two or more parton-parton scatterings within the same pp interaction [1]. In processes where a $\mathrm{W}$ and two jets are produced, the $x$ values are larger, $x \sim 10^{-2}$, and the parton densities are lower. However, a sizable contribution to double parton scattering (DPS) can still be expected if the second scattering, yielding two jets, occurs at a high rate. The study of DPS processes is important because it provides valuable information on the transverse distribution of partons in the proton [2] and on the multi-parton correlations in the hadronic wave function [3]. DPS also constitutes a background to new physics searches at the LHC [4, 5, 6].

This paper presents a study of DPS based on $\mathrm{W}+2$-jet events in pp collisions at $7 \mathrm{TeV}$. DPS with a $\mathrm{W}+2$-jet final state occurs when one hard interaction produces a $\mathrm{W}$ boson and another produces a dijet in the same pp collision. Events containing a $\mathrm{W}+2$-jet final state originating from single parton scattering (SPS) constitute an irreducible background.

\section{Effective cross section}

The effective cross section, $\sigma_{\text {eff }}$, is a measure of the transverse distribution of partons inside the colliding hadrons and their overlap in a collision. The effective cross section involves the cross section for two processes to occur simultaneously and the cross sections for the individual processes. If $\mathrm{A}$ and $\mathrm{B}$ are two independent processes, whose production cross sections are $\sigma_{\mathrm{A}}$ and $\sigma_{\mathrm{B}}$, respectively, $\sigma_{\mathrm{eff}}$ can be written as:

$$
\sigma_{\mathrm{eff}}=\frac{m}{2} \frac{\sigma_{\mathrm{A}} \cdot \sigma_{\mathrm{B}}}{\sigma_{\mathrm{A}+\mathrm{B}}^{\mathrm{DPS}}},
$$

where " $m$ " is a symmetry factor for indistinguishable $(m=1)$ and distinguishable $(m=2)$ finalstates and $\sigma_{\mathrm{A}+\mathrm{B}}^{\mathrm{DPS}}$ is the cross section of the two processes to occur simultaneously.

According to various phenomenological studies [7, 8, 9], the above cross sections should be inclusive. This requirement makes the determination of $\sigma_{\text {eff }}$ independent of the specific mechanisms of the first and second interactions, as well as of the parton distribution functions (PDF). However, in the present analysis an exclusive selection is performed by considering the events with one $\mathrm{W}$ boson and exactly two jets with $p_{T}>20 \mathrm{GeV} / \mathrm{c}$ and pseudorapidity, $\eta$, within \pm 2 . The pseudorapidity is defined as $\eta=-\ln [\tan (\theta / 2)]$, where $\theta$ is the polar angle measured with respect to the anti-clockwise beam direction. In the kinematic region of the present study, due to the requirement of having exactly 2 jets, the missing contribution of a larger number of parton scatterings is expected to be small and is estimated, with a sample of simulated events, to be less than $1 \%$ of the DPS contribution.

Assuming independent interactions from DPS, $\sigma_{\text {eff }}$ can be rewritten in terms of the cross sections at the stable particle level (defined as lifetime, $c \tau>10 \mathrm{~mm}$ ) within the detector acceptance. For the case of the $\mathrm{W}+2$-jet process one can define the fraction of DPS events as 
$f_{\mathrm{DPS}}=N_{\mathrm{W}+2 \mathrm{j}}^{\prime \mathrm{DPS}} / N_{\mathrm{W}+2 \mathrm{j}}^{\prime}$, where $N_{\mathrm{W}+2 \mathrm{j}}^{\prime}$ and $N_{\mathrm{W}+2 \mathrm{j}}^{\prime \mathrm{DPS}}$ are the yields of the of $\mathrm{W}$ bosons associated with two jets and associated with DPS, respectively. The effective cross section, $\sigma_{\text {eff }}$ can be reformulated as

$$
\sigma_{\mathrm{eff}}=\frac{\mathrm{R}}{f_{\mathrm{DPS}}} \cdot \sigma_{2 \mathrm{j}}^{\prime}
$$

where $R$ is the ration of the yield of $\mathrm{W}$ bosons associated with zero jets and the yield associated with DPS, $R=N_{\mathrm{W}+0 \mathrm{j}}^{\prime} / N_{\mathrm{W}+2 \mathrm{j}}^{\prime}$ and $\sigma_{2 \mathrm{j}}^{\prime}$ is the particle-level cross section for W-boson production for dijet events. Thus, the determination of the effective cross section reduces to a measurement of $R$, $\sigma_{2 \mathrm{j}}^{\prime}$, and $f_{\mathrm{DPS}}$.

For the extraction of the DPS fraction, $f_{\text {DPS }}$, observables that can discriminate between SPS and DPS are needed. For DPS events, the W and the dijet system are independent of each other, while for SPS events they are highly correlated. The present analysis uses the following observables:

- the relative $p_{T}$-balance between the two jets, $\Delta^{\text {rel }} p_{T}$, defined as:

$$
\Delta^{\mathrm{rel}} p_{T}=\frac{\left|\overrightarrow{p_{T}}\left(\mathrm{j}_{1}\right)+\overrightarrow{p_{T}}\left(\mathrm{j}_{1}\right)\right|}{\left|\overrightarrow{p_{T}}\left(\mathrm{j}_{1}\right)\right|+\left|\overrightarrow{p_{T}}\left(\mathrm{j}_{1}\right)\right|}
$$

Here $\overrightarrow{p_{T}}\left(\mathrm{j}_{1}\right)$ and $\overrightarrow{p_{T}}\left(\mathrm{j}_{2}\right)$ are the transverse momentum vectors of the leading (in $p_{T}$ ) and subleading jets. In DPS events, at leading order (LO), the two jets balance each other and $\Delta^{\mathrm{rel}} p_{T}$ is small, which is not the case for SPS events.

- The azimuthal angle between the W-boson and the dijet system, $\Delta S$, defined as:

$$
\Delta S=\arccos \left(\frac{\overrightarrow{p_{T}}\left(\mu, \mathrm{E}_{T}\right) \cdot \overrightarrow{p_{T}}\left(\mathrm{j}_{1}, \mathrm{j}_{2}\right)}{\left|\overrightarrow{p_{T}}\left(\mu, \mathrm{E}_{T}\right)\right| \cdot\left|\overrightarrow{p_{T}}\left(\mathrm{j}_{1}, \mathrm{j}_{2}\right)\right|}\right)
$$

where $\overrightarrow{p_{T}}\left(\mu, \mathrm{E}_{T}\right)$ and $\overrightarrow{p_{T}}\left(\mathrm{j}_{1}, \mathrm{j}_{2}\right)$ are the combined transverse momentum vectors of $\left(\mu, \mathbb{E}_{T}\right)$ and the two jets, respectively, with $\mathbb{E}_{T}$ as the missing transverse energy in the event, which is a measure of the transverse energy carried away by the neutrino from the W-boson decay. In DPS events, the W and dijet momentum vectors are randomly oriented, whereas in SPS events the $\mathrm{W}$ and the dijet momenta vectors tend to be back-to-back at LO.

\section{Unfolding and comparison with simulations}

The sample of $\mathrm{W}+2$-jet events is selected as discussed in the previous section. The contributions of all backgrounds are subtracted from the data distributions before unfolding.

The distributions of the DPS-sensitive observables for the selected events are corrected for selection efficiencies and detector effects. The shape of the distribution of $\Delta^{\text {rel }} p_{T}$ and $\Delta S$ is more important than the absolute normalization in the extraction of the DPS fraction. Therefore, the unfolding is carried out for the shapes of the $\Delta^{\text {rel }} p_{T}$ and $\Delta S$ distributions. The measured distributions are unfolded to the level of stable particles within the folowing phase space:

$$
\begin{array}{r}
1 \not \mu: p_{T}>35 \mathrm{GeV} \text { and }|\eta|<2.1, \\
\mathrm{E}_{T}>30 \mathrm{GeV} \text { and } M_{T}>50 \mathrm{GeV} / \mathrm{c}^{2},
\end{array}
$$

Exactly 2 jets: $p_{T}>20 \mathrm{GeV} / \mathrm{c}$ and $|\eta|<2.0$. 
Various simulations at particle level are compared with the fully corrected DPS-sensitive observables:

- MadGraph 5 + Pythia 8: W+ jets events are generated by means of MAdGraPh 5 $[10,11]$ followed by hadronization and parton showering using the $4 \mathrm{C}$ tune [12] of PYTHIA 8 [13]. The MPI [1] are simulated with the PYTHIA 8 event generator.

- Powheg 2 + Pythia 6: $\mathrm{W}+2$-jet events are also produced up to NLO accuracy with the Powheg 2 [14] event generator with the "Multi-scale improved NLO" (MiNLO) method [15]. Hadronization and parton showering is carried out with Pyтнia 6 [16], tune Z2* [17].

- Pythia 8: $\mathrm{W}+$ jets events are generated with the $4 \mathrm{C}$ tune of the Pythia 8 event generator, which produces hard subprocesses with a $\mathrm{W}$ boson and either zero or one additional parton in the final state. It also performs hadronization and parton showering.

A comparison of various simulations for inclusive $\mathrm{W}$ production with the corrected distributions is shown in Fig. 1.
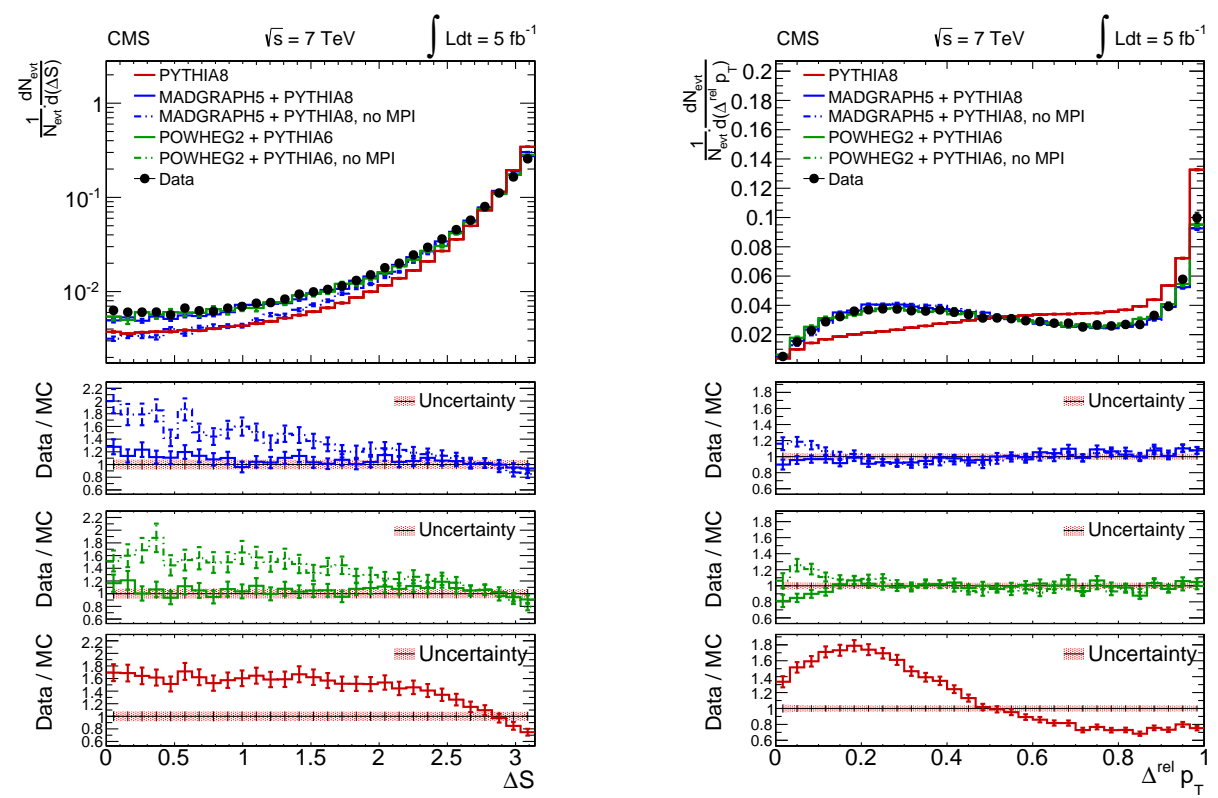

Figure 1: Fully corrected data distributions, normalized to unity, for the DPS-sensitive observables $\Delta^{\text {rel }} p_{T}$ and $\Delta S$ together with the several Monte Carlo predictions as described in the text.

The $\Delta^{\text {rel }} p_{T}$ and $\Delta S$ distributions are properly described by MADGRAPH $5+$ PYTHIA 8 . The NLO predictions for $\mathrm{W}+2$-jet production obtained with POWHEG $2+$ PYTHIA 6 also satisfactorily describe the data. The PYTHIA 8 simulation underestimates the measurements. This discrepancy is due to the fact that PутнIA 8 generates only $2 \rightarrow 1$ and $2 \rightarrow 2$ processes and most of the additional jets are produced during parton showering, and have a softer $p_{T}$ spectrum than that measured in data. The difference is mainly in the DPS-sensitive region. Therefore, event generators used to define SPS backgrounds must include a proper implementation of additional hard radiation. If it is not included, the effect of missing hard radiation might be interpreted as a DPS contribution. Without MPI, the LO and NLO predictions from MADGRAPH 5 + PythiA 8 and Powheg $2+$ Pythia 6 are unable to describe the data shown in Fig. 1. 


\section{Determination of the effective cross section}

As discussed in Section 2 to calculate the effective cross section the measurements of DPS fraction, the measurements of the dijet cross section and $R$ are necessary.

The fraction of $\mathrm{W}+2$-jet events produced by DPS is extracted by performing a template fit using the signal and background templates to the fully corrected distributions of $\Delta^{\text {rel }} p_{T}$ and $\Delta S$. The signal template is obtained by randomly mixing independently produced $\mathrm{W}$ and dijet events, whereas the background template is produced from the $\mathrm{W}+2$-jet sample simulated with MADGRAPH 5 + PYTHIA 8, in which events with MPI-tagged partons within the acceptance $(|\eta|<$ $2)$ are removed. The fitted value of the DPS fraction $\left(f_{\text {DPS }}\right)$ is:

$$
f_{\mathrm{DPS}}=0.055 \pm 0.002 \text { (stat.) } \pm 0.014 \text { (syst.). }
$$

The ratio, $R$, of the yield of events with a $\mathrm{W}$ boson in the final state and no jets to the yield of events with a $\mathrm{W}$ boson and exactly two jets with $p_{T}>20 \mathrm{GeV} / \mathrm{c}$ and $|\eta|<2.0$ is measured to be

$$
R=27.8 \pm 0.2 \text { (stat.) } \pm 3.3 \text { (syst.). }
$$

The cross section for events with exactly two jets with $p_{T}>20 \mathrm{GeV} / \mathrm{c}$ and $|\eta|<2.0$ is

$$
\sigma_{2 \mathrm{j}}^{\prime}=0.0409 \pm 0.0004 \text { (stat.) } \pm 0.0061 \text { (syst.) mb. }
$$

With the values of $f_{\mathrm{DPS}}, R$, and $\sigma_{2 \mathrm{j}}^{\prime}$ in eq.(2.1), the effective cross section is determined to be:

$$
\sigma_{\text {eff }}=20.7 \pm 0.8 \text { (stat.) } \pm 6.6 \text { (syst.) mb. }
$$

Figure 2 shows a comparison of the effective cross sections obtained using different processes at various centre-of-mass energies. From the experimental results, a firm conclusion on the energy dependence of $\sigma_{\text {eff }}$ cannot be drawn because of the large systematic uncertainties. The CMS measurement is consistent with previous measurements performed at the Tevatron and by the ATLAS Collaboration at the LHC. The CMS measurement is also consistent with predictions from PYTHIA of 20-30 mb, depending on the tune.

\section{References}

[1] T. Sjöstrand and M. Van Zijl, "A Multiple Interaction Model for the Event Structure in Hadron Collisions", Phys. Rev. D 36 (1987) 2019, doi:10.1103/PhysRevD.36.2019.

[2] M. Diehl, D. Ostermeier, and A. Schäfer, "Elements of a theory for multiparton interactions in QCD", JHEP 03 (2012) 89, doi:10.1007/JHEP03(2012)089, arXiv:1111.0910.

[3] G. Calucci and D. Treleani, "Disentangling correlations in Multiple Parton Interactions", Phys. Rev. D 83 (2011) 016012, doi:10.1103/PhysRevD.83.016012, arXiv:1009.5881.

[4] A. D. Fabbro and D. Treleani, "A double parton scattering background to Higgs boson production at the LHC", Phys. Rev. D 61 (2000) 077502, doi:10.1103/PhysRevD.61.077502, arXiv:hep-ph/9911358.

[5] M. Y. Hussein, "A double parton scattering background to associate $\mathrm{WH}$ and $\mathrm{ZH}$ production at the LHC", Nucl. Phys. Proc. Suppl. 174 (2007) 55, doi:10.1016/j.nuclphysbps.2007.08.086, arXiv:hep-ph/0610207. 


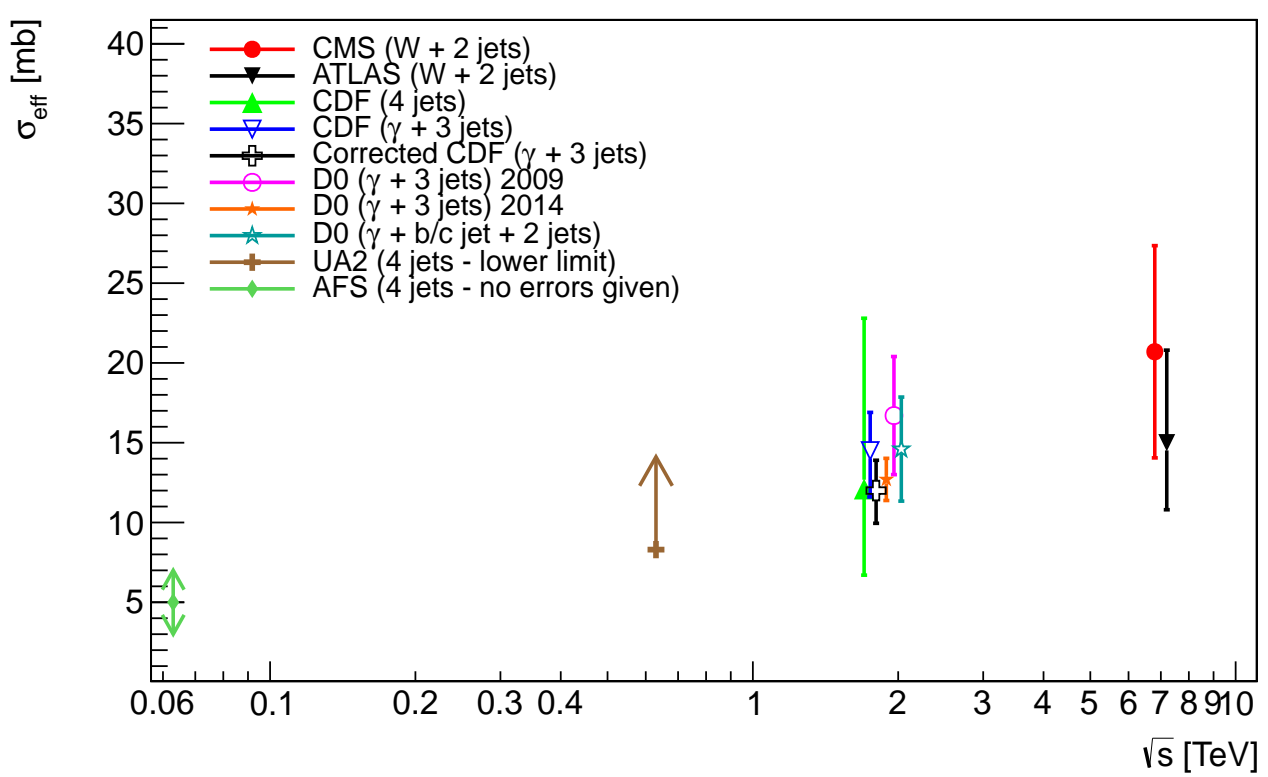

Figure 2: Centre-of-mass energy dependence of $\sigma_{\text {eff }}$ measured by different experiments using different processes.

[6] D. Bandurin, G. Golovanov, and N. Skachkov, "Double parton interactions as a background to associated HW production at the Tevatron", JHEP 04 (2011) 054, doi:10.1007/JHEP04(2011)054, arXiv:1011.2186.

[7] D. Treleani, "Double parton scattering, diffraction and effective cross section", Phys. Rev. D 76 (2007) 076006, doi:10.1103/PhysRevD.76.076006, arXiv:0708.2603.

[8] M. Bähr, M. Myska, M. H. Seymour, and A. Siodmok, "Extracting $\sigma_{\text {eff }}$ from the CDF $\gamma+3$ jets measurement", JHEP 03 (2013) 129, doi:10.1007/JHEP03(2013)129, arXiv:1302.4325.

[9] M. H. Seymour and A. Siodmok, "Extracting $\sigma_{\text {eff }}$ from the LHCb double-charm measurement", (2013). arXiv:1308.6749.

[10] J. Alwall et al., "MADGRAPH 5: going beyond", JHEP 06 (2011) 128, doi:10.1007/JHEP06(2011)128, arXiv:1106.0522.

[11] F. Maltoni and T. Stelzer, "MadEvent: Automatic event generation with MADGRAPH", JHEP 02 (2003) 027, doi:10.1088/1126-6708/2003/02/027, arXiv:hep-ph/0208156.

[12] R. Corke and T. Sjöstrand, "Interleaved parton showers and tuning prospects", JHEP 03 (2011) 032, doi:10.1007/JHEP03(2011)032, arXiv:1011.1759.

[13] T. Sjöstrand, S. Mrenna, and P. Z. Skands, "A brief introduction to Pythia 8.1", Comput. Phys. Commun. 178 (2008) 852, doi:10.1016/j.cpc.2008.01.036, arXiv:0710.3820.

[14] K. Hamilton and P. Nason, "Improving NLO-parton shower matched simulations with higher order matrix elements", JHEP 06 (2010) 039, doi:10.1007/JHEP06(2010)039, arXiv:1004.1764.

[15] K. Hamilton, P. Nason, and G. Zanderighi, "MINLO: multi-scale improved NLO”, JHEP 10 (2012) 155, doi:10.1007/JHEP10(2012)155, arXiv:1206.3572.

[16] T. Sjöstrand, S. Mrenna, and P. Z. Skands, "PYTHIA 6.4 physics and manual”, JHEP 05 (2006) 026, doi:10.1088/1126-6708/2006/05/026, arXiv:hep-ph/0603175.

[17] CMS Collaboration, "Study of the underlying event at forward rapidity in pp collisions at $\sqrt{s}=0.9$, 2.76, and 7 TeV", JHEP 04 (2013) 072, doi:10.1007/JHEP04(2013)072, arXiv:1302.2394. 